\title{
TeroKit: A Database-driven Web Server for Terpenome Research
}

Tao Zeng ${ }^{1, \#}$, Zhihong Liu ${ }^{2, \#}$, Jingyuan Zhuang ${ }^{1}$,Yitao Jiang ${ }^{1}$, Wengan $\mathrm{He}^{1}$, Hongjuan Diao $^{1}$, Nan $\mathrm{Lv}^{1}$, Yongxing Jian ${ }^{1}$, Danhong Liang ${ }^{1}$, Yufan Qiu ${ }^{1}$, Rong Zhang ${ }^{1}$, Fan Zhang ${ }^{1}$, Xiaowen Tang ${ }^{1}$, Ruibo $\mathrm{Wu}^{1, *}$

${ }^{1}$ Guangdong Provincial Key Laboratory of New Drug Design and Evaluation, School of Pharmaceutical Sciences, Sun Yat-sen University, Guangzhou 510006, People's Republic of China

${ }^{2}$ State Key Laboratory of Applied Microbiology Southern China, Guangdong Provincial Key Laboratory of Microbial Culture Collection and Application, Guangdong Open Laboratory of Applied Microbiology, Guangdong Institute of Microbiology, Guangdong Academy of Sciences, Guangzhou 510070, China

${ }^{\#}$ T. Zeng and Z. Liu contributed equally to this work.

*E-mail: wurb3@mail.sysu.edu.cn

Table S1 S5 and Figure S1 S3 
Table S1. Distribution of terpenome in public database.

\begin{tabular}{ccl}
\hline Database & Identified terpenome & \multicolumn{1}{c}{ Website } \\
\hline CAS & 71100 & https://scifinder.cas.org/ \\
PubChem & 57593 & https://pubchem.ncbi.nlm.nih.gov/ \\
ChEMBL & 17586 & https://www.ebi.ac.uk/chembl/ \\
BindingDB & 1846 & http://www.bindingdb.org \\
KEGG & 1913 & https://www.kegg.jp/ \\
DrugBank & 157 & https://www.drugbank.ca/ \\
ZINC & 33042 & http://zinc.docking.org/ \\
PDB & 261 & https://www.rcsb.org/ \\
CSD & 4653 & https://www.ccdc.cam.ac.uk/ \\
\hline
\end{tabular}


Table S2. List of 56 journals consulted in the statistics analysis

\section{Journal Name}

Acta chemica Scandinavica, Acta crystallographica, Agricultural and biological chemistry, Applied and environmental microbiology, Asian Pacific journal of tropical medicine, Biochemical systematics and ecology, Biological \& pharmaceutical bulletin, Bioorganic \& medicinal chemistry, Bioorganic \& medicinal chemistry letters, Bioscience, biotechnology, and biochemistry, Applied microbiology and biotechnology, BMC complementary and alternative medicine, $B M C$ research notes, Canadian journal of microbiology, Carbohydrate research, Chemical \& pharmaceutical bulletin, Chemistry \& biodiversity, Chemistry, Drugs, European journal of organic chemistry, Evidence based complementary and alternative medicine, Fitoterapia, Food chemistry, Helvetica chimica acta, Inflammopharmacology, Journal of agricultural and food chemistry, The Journal of antibiotics, Journal of Asian natural products research, Journal of ethnopharmacology, Journal of natural products, The Journal of organic chemistry, Journal of the American Oil Chemists' Society, Malaria journal, Marine drugs, Molecules, Natural product communications, Natural product letters, Natural product research, Organic and medicinal chemistry letters, Organic letters, Parasitology research, Pharmaceutical biology, Die Pharmazie, Phytochemical analysis, Phytochemistry, Phytochemistry letters, Phytomedicine: international journal of phytotherapy and phytopharmacology, Phytotherapy research, Planta, Planta medica, Pure and applied chemistry, Steroids, Tetrahedron, Tetrahedron letters, China journal of Chinese materia medica, Journal of Chinese medicinal materials 
Table S3. The list of physicochemical properties calculated in TeroKit Property Description

Tool

$\begin{array}{ccc}\text { Alogp } & \begin{array}{c}\text { Ghose-Crippen oil-water partition } \\ \text { coefficient }\end{array} & \text { Padel-Descriptor } \\ \text { FSp3 } & \text { fraction of Csp3 atoms } \\ \text { N_Rb } & \text { Number of rotatable bonds } & \text { Padel-Descriptor } \\ \text { N_Acid } & \text { Number of acidic groups } & \text { Padel-Descriptor } \\ \text { N_Base } & \text { Number of basic groups } & \text { Padel-Descriptor } \\ \text { N_Aro } & \text { Number of aromatic atoms } & \text { Padel-Descriptor } \\ \text { N_Ring } & \text { Number of rings } & \text { Padel-Descriptor } \\ \text { NHa } & \text { number of hydrogenbond acceptions } & \text { Padel-Descriptor } \\ \text { NHd } & \text { number of hydrogenbond donors } & \text { Padel-Descriptor } \\ \text { TPSA } & \text { topological polar surface area } & \text { Padel-Descriptor } \\ \text { nChiral } & \text { Number of chiral centers } & \text { Padel-Descriptor }\end{array}$


Table S4. The list of ADMET properties in TeroKit

\section{Property}

Passive Intestinal Absorption

Blood Brain Barrier Penetration

CYP450 2D6 inhibitor

Biodegradation

Ames test

Developmental Toxicity Potential
Model

Public models

Public models

TOPKAT models

TOPKAT models

TOPKAT models

TOPKAT models 
Table S5. Tools used in TeroKit

\begin{tabular}{|c|c|c|}
\hline Tools & Purpose & Link \\
\hline DNP & Structure data source & $\begin{array}{l}\text { dnp.chemnetbase.com/faces/chemical/C } \\
\text { hemicalSearch.xhtml }\end{array}$ \\
\hline PubChem & Structure data source & pubchem.ncbi.nlm.nih.gov/ \\
\hline ChEMBL & $\begin{array}{l}\text { Bioactive molecules } \\
\text { data source }\end{array}$ & www.ebi.ac.uk/chembl/ \\
\hline BindingDB & $\begin{array}{l}\text { Bioactive molecules } \\
\text { data source }\end{array}$ & $\begin{array}{l}\text { http://www.bindingdb.org/bind/index.js } \\
\mathrm{p}\end{array}$ \\
\hline DrugBank & $\begin{array}{l}\text { Cross-link of } \\
\text { molecules }\end{array}$ & https://www.drugbank.ca/ \\
\hline KEGG & $\begin{array}{l}\text { Cross-link of } \\
\text { molecules }\end{array}$ & https://www.kegg.jp/ \\
\hline $\mathrm{CCDC}$ & $\begin{array}{l}\text { Cross-link of } \\
\text { molecules }\end{array}$ & https://www.ccdc.cam.ac.uk/ \\
\hline PDB & $\begin{array}{l}\text { Cross-link of } \\
\text { molecules }\end{array}$ & https://www.rcsb.org/ \\
\hline ZINC & $\begin{array}{l}\text { Vendors and cross- } \\
\text { link of molecules }\end{array}$ & http://zinc.docking.org/ \\
\hline RDKit & $\begin{array}{l}\text { Conformer } \\
\text { generating and } \\
\text { structure processing }\end{array}$ & www.rdkit.org/ \\
\hline OpenBabel & $\begin{array}{l}\text { Chemical file format } \\
\text { convertion }\end{array}$ & openbabel.org/ \\
\hline $\begin{array}{l}\text { PaDEL- } \\
\text { Descriptor }\end{array}$ & $\begin{array}{l}\text { Physicochemical } \\
\text { properties calculation }\end{array}$ & $\begin{array}{l}\text { padel.nus.edu.sg/software/padeldescript } \\
\text { or }\end{array}$ \\
\hline Pipeline Pilot & $\begin{array}{l}\text { ADMET properties } \\
\text { calculation }\end{array}$ & $\begin{array}{l}\text { https://www.3dsbiovia.com/products/col } \\
\text { laborative-science/biovia-pipeline-pilot/ }\end{array}$ \\
\hline $\begin{array}{l}\text { ChemDoodle } \\
\text { Web }\end{array}$ & $\begin{array}{l}\text { Structure draw and } \\
\text { display }\end{array}$ & https://web.chemdoodle.com/ \\
\hline Cytoscape.js & Network display & https://js.cytoscape.org/ \\
\hline JQuery & $\begin{array}{l}\text { Foregroud and } \\
\text { background } \\
\text { interaction }\end{array}$ & https://jquery.com/ \\
\hline
\end{tabular}




\begin{tabular}{l|l|l}
\hline PostgreSQL & Storage database & https://www.postgresql.org/ \\
\hline Bingo & $\begin{array}{l}\text { Molecular search } \\
\text { cartridge }\end{array}$ & $\begin{array}{l}\text { https://lifescience.opensource.epam.co } \\
\mathrm{m} / \text { bingo/index.html }\end{array}$ \\
\hline Golang & Web server language & https://golang.org/ \\
\hline
\end{tabular}



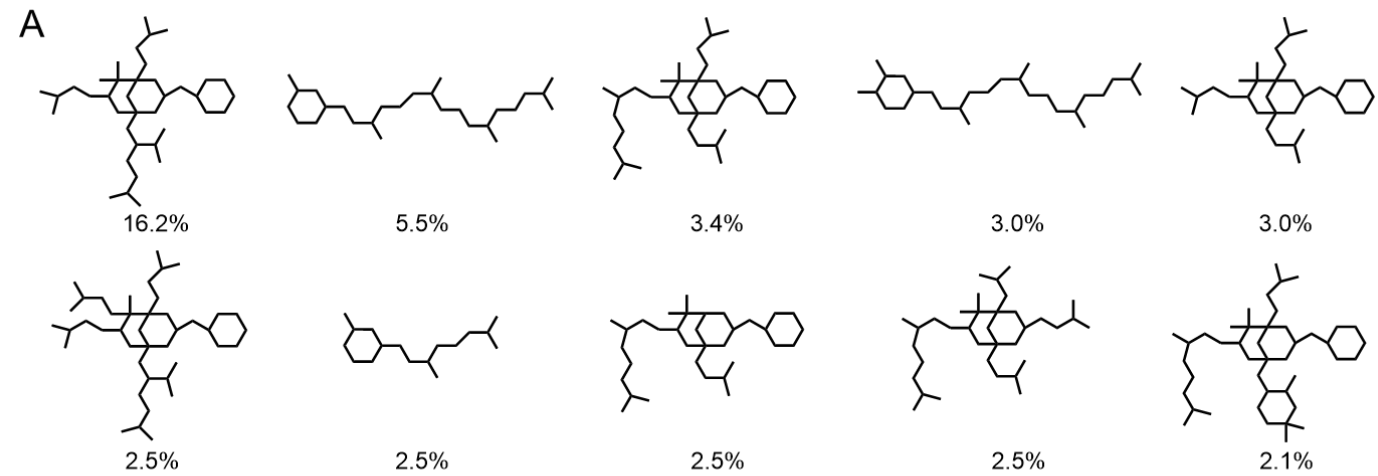

B
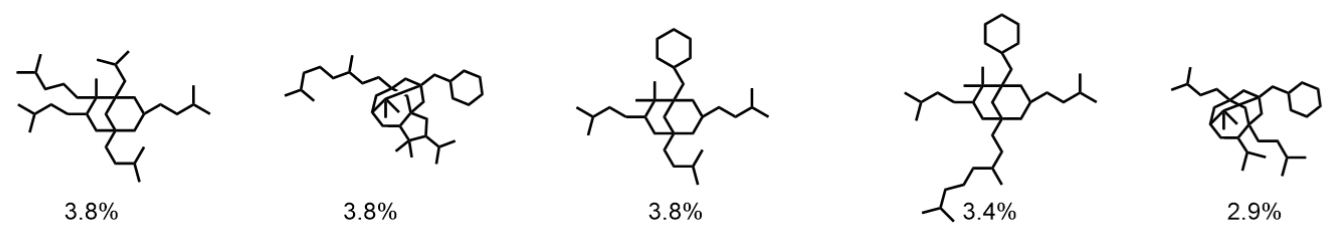<smiles>CCC(C)CCC(C)CCC(C)C</smiles>
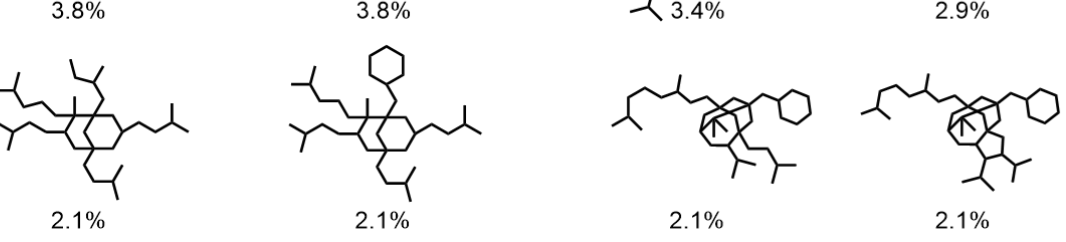

Figure S1. Top 10 skeletons and their proportions in Hypericaceae (A) and Clusiaceae (B) family. 

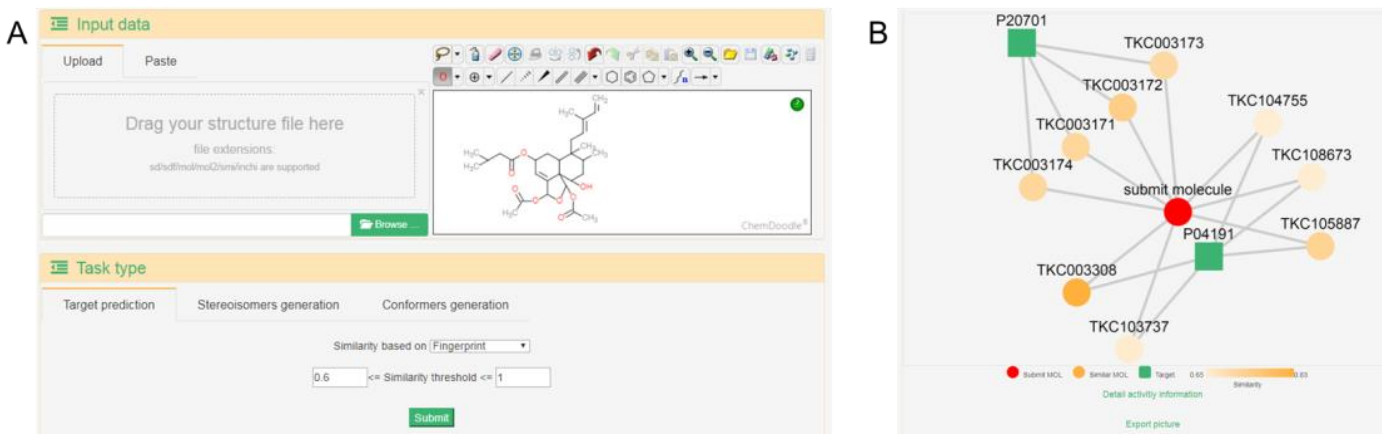

Figure S2. The candidate structure (A) and the target map of similar compounds (B). The structure was obtained from a recent study ${ }^{1}$ which illustrated its cytotoxicity to cancer cells. And the target map we got also suggested that the structure could target to P04191 (UniProtKB), which is called sarcoplasmic/endoplasmic reticulum calcium ATPase 1 (SERCA) and can be a promising target for cancer therapy. ${ }^{2}$ 


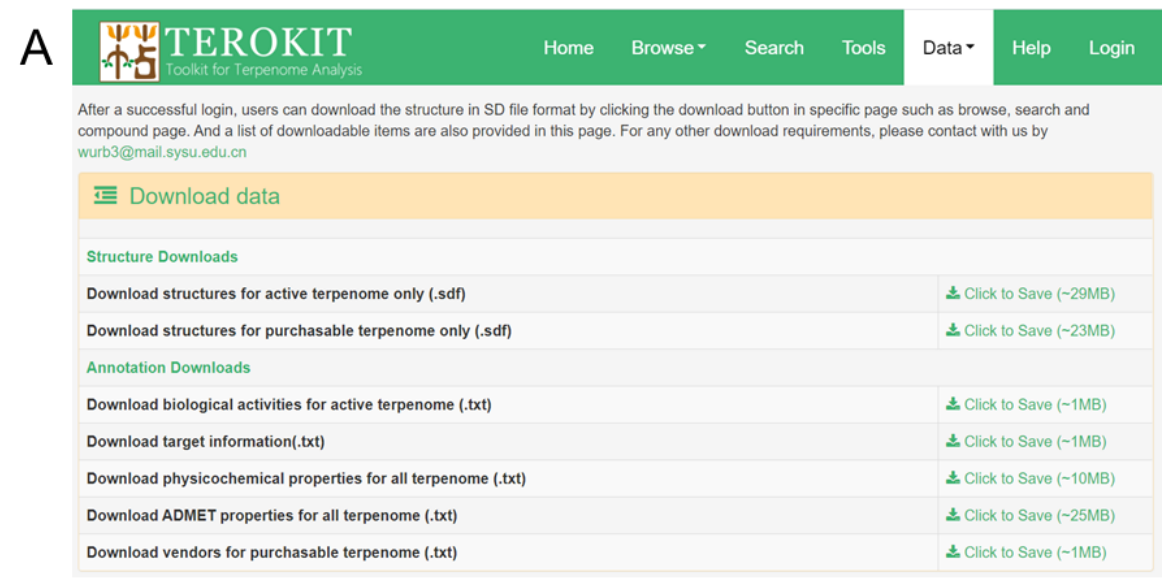

B $\quad$ Home Browse- Search Tools Data- Help Login

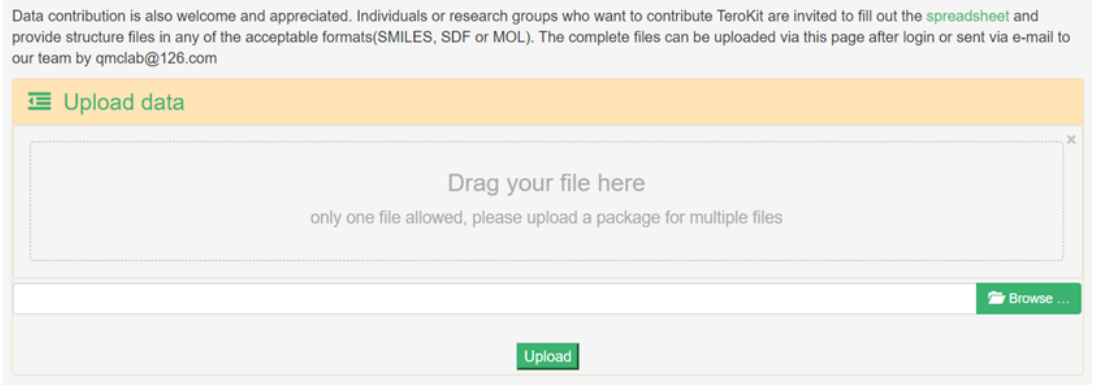

Figure S3. The download page (A) and upload page (B) of TeroKit.

\section{Reference}

1. Liu, F.; Ma, J.; Shi, Z.; Zhang, Q.; Wang, H.; Li, D.; Song, Z.; Wang, C.; Jin, J.; Xu, J.; Tuerhong, M.; Abudukeremu, M.; Shuai, L.; Lee, D.; Guo, Y., Clerodane Diterpenoids Isolated from the Leaves of Casearia Graveolens. J. Nat. Prod. 2020, 1, 36-44.

2. Denmeade, S. R.; Isaacs, J. T., The Serca Pump as a Therapeutic Target: Making a "Smart Bomb" for Prostate Cancer. Cancer Biol. Ther. 2005, 4, 21-29. 